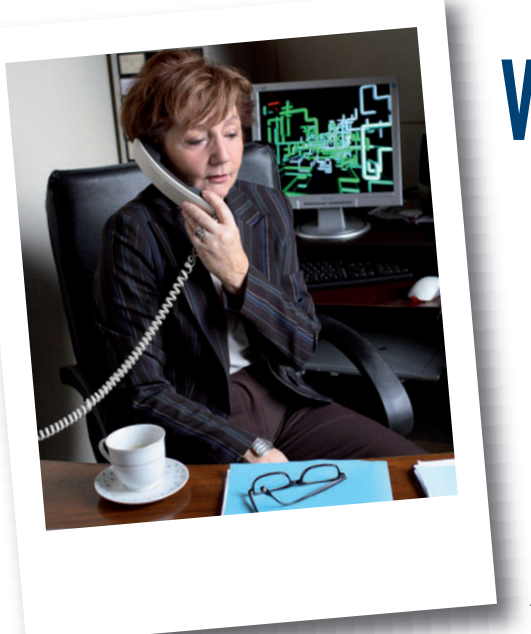

\title{
Vers une fédération des sociétés scientifiques autour des sciences physiques
}

Un constat s'impose : la SFP est une société savante héritière d'une tradition prestigieuse, mais qui a toujours été et demeure petite, malgré les efforts divers de ses directions successives. II est clair qu'elle n'atteint pas la taille critique pour faire entendre son point de vue dans un monde universitaire en profonde mutation où, de plus, la recherche et l'innovation vont maintenant de pair. C'est d'ailleurs pour cette raison que la SFP s'est récemment rapprochée des sociétés de mathématiques et de chimie pour apporter des contributions aux débats suscités autour des réformes ministérielles. Aujourd'hui, il paraît important de tenter un regroupement de la SFP avec d'autres sociétés savantes dans les domaines faisant partie ou proches des sciences physiques (l'optique, l'électronique, la mécanique, le vide, l'acoustique, etc). Ces sociétés sont nombreuses et rassemblent des chercheurs et surtout des ingénieurs.

C'est ainsi qu'est née l'idée de créer une Fédération Française des Sociétés Scientifiques (FS2), suivant en cela l'exemple de la Fédération Française pour les Sciences de la Chimie (FFC). Au départ, elle devrait regrouper la SFP, la SFO (Société Française d'Optique) et la SEE (Société de l'Électricité, de l'Électronique et des Technologies de l'Information et de la Communication). Clairement, d'autres sociétés savantes pourraient rapidement rallier la FS2, de façon à rassembler la large communauté qui travaille dans le domaine des sciences exactes, depuis la recherche la plus fondamentale jusqu'aux applications industrielles. Des statuts, impliquant un minimum d'administration, sont en cours d'élaboration. Le budget de cette fédération, au départ modeste et fourni par les sociétés constituantes, devrait rapidement augmenter en fonction de l'aide de mécènes qui seront sollicités.

Les trois sociétés fondatrices souhaitent, en se fédérant, mettre en commun leur vision pour devenir une force de proposition écoutée dans la société de la connaissance d'aujourd'hui. Elles sont, en effet, directement concernées par les défis majeurs de notre temps, tels la raréfaction de l'énergie, la sécurité des citoyens, le réchauffement de la Terre, l'hyperdéveloppement des plateformes urbaines ou l'avènement de la société de l'immatériel. Face à ces questions, la fédération, forte de ses compétences complémentaires, devrait pouvoir apporter des éléments de solution utiles en multipliant les éclairages et les avis, tout en fournissant des espaces d'échange.

Plusieurs types d'actions communes dans différents domaines sont envisagés : création de prix communs, organisation de colloques à haute visibilité sur des sujets de société, contacts avec les média, analyses et prises de position communes auprès des instances décisionnelles, promotion de l'enseignement des sciences et des carrières techniques. II reste donc à convaincre les mécènes de l'intérêt d'une telle fédération, de façon à pouvoir la faire vivre à long terme, sans diminuer l'influence de chaque société constituante sur le milieu qu'elle représente traditionnellement. La SFP doit trouver son équilibre dans ce nouveau contexte. Les enjeux justifient, me semble-t-il, cet effort d'adaptation au monde actuel. 